\title{
The HII region G35.673-00.847: another case of triggered star formation?
}

\author{
S. Paron ${ }^{1,2}$, A. Petriella ${ }^{1}$, and M. E. Ortega ${ }^{1}$ \\ ${ }^{1}$ Instituto de Astronomía y Física del Espacio (IAFE), CC 67, Suc. 28, 1428 Buenos Aires, Argentina \\ e-mail: sparon@iafe.uba.ar \\ 2 FADU - Universidad de Buenos Aires, Argentina
}

Received 30 June 2010 / Accepted 18 October 2010

\begin{abstract}
Aims. As part of a systematic study that we are performing to increase the observational evidence of triggered star formation in the surroundings of HII regions, we analyze the ISM around the HII region G35.673-00.847, a poorly studied source.

Methods. Using data from the large-scale surveys Two Micron All Sky Survey, Galactic Legacy Infrared Mid-Plane Survey Extraordinaire (GLIMPSE), MIPSGAL, Galactic Ring Survey (GRS), VLA Galactic Plane Survey (VGPS), and NRAO VLA Sky Survey (NVSS), we performed a multiwavelength study of G35.673-00.847 and its surroundings.

Results. The mid-IR emission shows that G35.673-00.847 has an almost semi-ring like shape with a cut towards the galactic west. The radius of this semi-ring is about 1..5 $(\sim 1.6 \mathrm{pc}$, at the distance of $\sim 3.7 \mathrm{kpc})$. The distance was estimated from an HI absorption study and the analysis of the molecular gas. We find a molecular shell composed of several clumps distributed around the HII region, suggesting that its expansion is collecting the surrounding material. We identify several YSO candidates across the molecular shell. Finally, comparing the HII region dynamical age and the fragmentation time of the molecular shell, we discard the so-called collect and collapse as being the mechanism responsible for the YSO formation, suggesting that other processes such as radiative-driven implosion and/or small-scale Jeans gravitational instabilities operate.
\end{abstract}

Key words. HII regions - ISM: clouds - stars: formation

\section{Introduction}

In the past few years, the Galactic Legacy Infrared Mid-Plane Survey Extraordinaire (GLIMPSE) performed with data obtained from the Spitzer Space Telescope has been a very useful tool for studying the Galactic IR emission with unprecedented quality and resolution, and still remains so. Using these midIR data, it is possible for instance, to clearly identify the photodissociation regions (PDRs) surrounding HII regions. Thus, from a multiwavelength analysis we can study the interaction between the HII region and the surrounding interstellar medium (ISM) and identify triggered star formation. One of the triggered processes that has been widely studied in HII region borders is the "collect and collapse", which was originally proposed by Elmegreen \& Lada (1977). This process occurs during the supersonic expansion of an HII region when a dense layer of material is collected between the ionization and the shock fronts. When this layer fragments into massive condensations that can then collapse to promote the formation of new massive stars and/or clusters. Observational studies have found evidence that this mechanism is taking place in several HII regions (see e.g. Petriella et al. 2010; Pomarès et al. 2009; Zavagno et al. 2007, and references therein).

G35.673-00.847 (hereafter G35.6) is a poorly studied HII region. The source was cataloged in the HII region catalogue of Lockman (1989), who obtained a recombination line at $v_{\mathrm{LSR}} \sim$ $60 \mathrm{~km} \mathrm{~s}^{-1}$. According to the IRAS Point Source Catalog, G35.6 coincides with the source IRAS 18569+0159. In the NRAO VLA Sky Survey (NVSS), Condon et al. (1998) identified two radio sources, NVSS $185929+020334$ and $185938+020012$, towards this region.
This work is part of a systematic study that we are performing to increase the observational evidence of triggered star formation in the surroundings of HII regions. We present a molecular and near- and mid-IR study of the environment that surrounds the HII region G35.6 to explore the ISM around it, and look for signatures of star formation.

\section{Data}

We analyzed data extracted from the four large-scale surveys Two Micron All Sky Survey (2MASS) $)^{1}$, Galactic Legacy Infrared Mid-Plane Survey Extraordinaire (GLIMPSE), MIPSGAL, and GRS ${ }^{2}$.

GLIMPSE is a mid-infrared survey of the inner Galaxy performed using the Spitzer Space Telescope. We used the mosaicked images from GLIMPSE and the GLIMPSE Point-Source Catalog (GPSC) acquired by Spitzer-IRAC (3.6, 4.5, 5.8 and $8 \mu \mathrm{m})$. IRAC has an angular resolution of between 1".5 and 1".9 (see Fazio et al. 2004; Werner et al. 2004). MIPSGAL is a survey of the same region as GLIMPSE, using MIPS instrument (24 and $70 \mu \mathrm{m}$ ) on Spitzer. The MIPSGAL resolution at $24 \mu \mathrm{m}$ is 6". The GRS was performed by Boston University and the Five College Radio Astronomy Observatory (FCRAO). The survey maps the Galactic ring in the ${ }^{13} \mathrm{CO} J=1-0$ line with an angular and spectral resolution of $46^{\prime \prime}$ and $0.2 \mathrm{~km} \mathrm{~s}^{-1}$, respectively

\footnotetext{
${ }^{1}$ 2MASS is a joint project of the University of Massachusetts and the Infrared Processing and Analysis Center/California Institute of Technology, funded by the National Aeronautics and Space Administration and the National Science Foundation.

${ }^{2}$ Galactic Ring Survey (Jackson et al. 2006).
} 

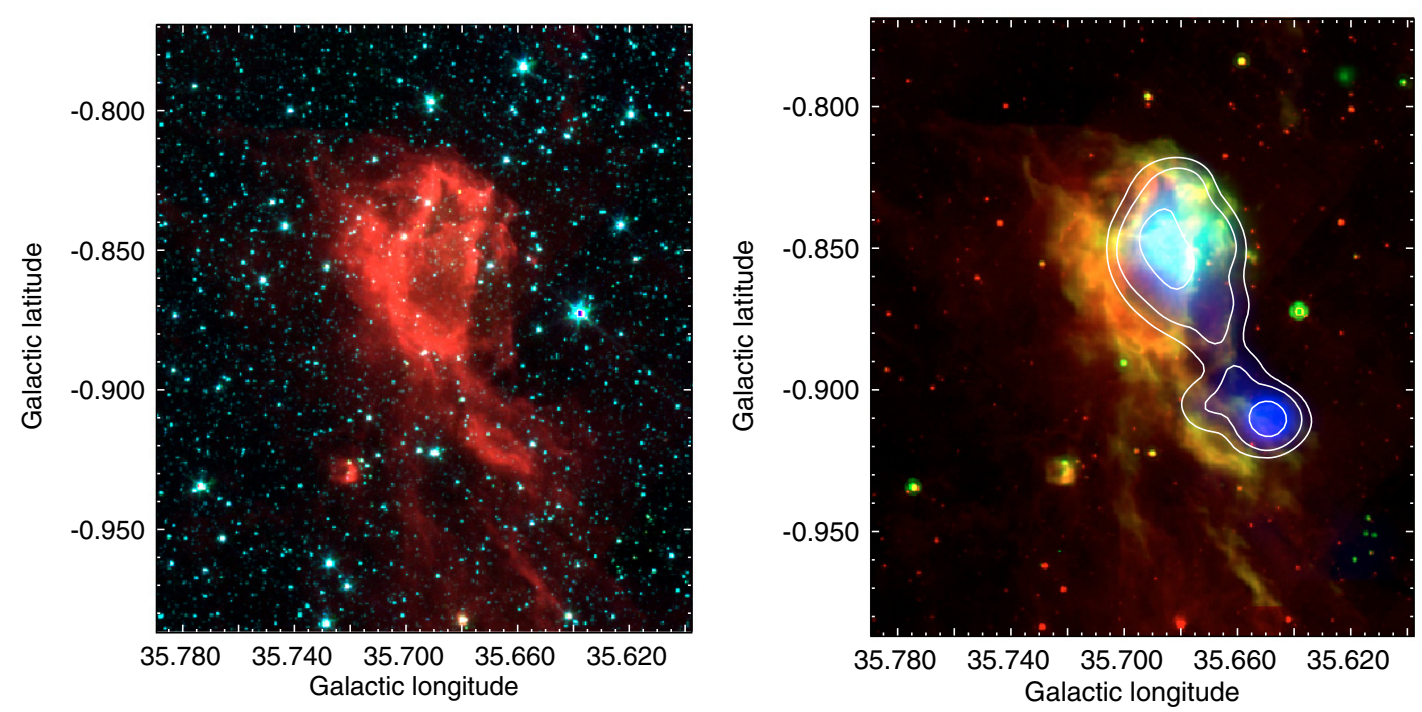

Fig. 1. Left: Spitzer-IRAC three-color image ( $3.6 \mu \mathrm{m}=$ blue, $4.5 \mu \mathrm{m}=$ green, and $8 \mu \mathrm{m}=$ red). Right: color composite image where the SpitzerIRAC $8 \mu \mathrm{m}$ emission is displayed in red, the Spitzer-MIPSGAL emission at $24 \mu \mathrm{m}$ is shown in green, and the NVSS radio continuum emission at $20 \mathrm{~cm}$ is presented in blue and emphasized by white contours with levels of $2.5,6$, and $20 \mathrm{mJy} \mathrm{beam}^{-1}$. The $\sigma_{\mathrm{rms}}$ of the NVSS data is $0.45 \mathrm{mJy}_{\text {beam }}{ }^{-1}$.

(see Jackson et al. 2006). The observations were performed in both position-switching and on-the-fly mapping modes, achieving an angular sampling of $22^{\prime \prime}$.

In addition we used HI data with an angular resolution of $\sim 1^{\prime}$ extracted from the VLA Galactic Plane Survey (VGPS; Stil et al. 2006) and radio continuum data extracted from the NRAO VLA Sky Survey (NVSS) with an angular resolution of $\sim 45^{\prime \prime}$ (Condon et al. 1998).

\section{Presentation of G35.673-00.847 (G35.6)}

Figure 1 shows two composite three-color images of G35.6. The left image displays three Spitzer-IRAC bands: $3.6 \mu \mathrm{m}$ (in blue), $4.5 \mu \mathrm{m}$ (in green), and $8 \mu \mathrm{m}$ (in red). The right image shows the Spitzer-IRAC emission at $8 \mu \mathrm{m}$ (in red), the Spitzer-MIPSGAL emission at $24 \mu \mathrm{m}$ (in green), and the NVSS radio continuum emission at $20 \mathrm{~cm}$ (in blue and emphasized with white contours). Both figures clearly show the PDR visible in the $8 \mu \mathrm{m}$ emission, which originates mainly in the polycyclic aromatic hydrocarbons (PAHs). The PAH emission delineates the HII region boundaries because these large molecules are destroyed inside the ionized region, but are excited in the PDR by the radiation leaking from the HII region (Pomarès et al. 2009). The $24 \mu \mathrm{m}$ emission reveals the presence of hot dust, and the radio continuum emission shows two sources, one related to G35.6, probably due to its ionized gas, and another lying towards the south. These sources are NVSS 185929+020334 and 185938+020012, respectively (Condon et al. 1998). The PAH emission shows that G35.6 has an almost semi-ring like shape with a cut towards the Galactic west. The radius of this semi-ring is about 1'.5. Extending towards the south is another visible PDR, which can be related to the radio continuum source NVSS $185938+020012$. On the other hand, a little bubble is present in the field, at $l=35^{\circ} .722, b=-0.928$. The emission at $8 \mu \mathrm{m}$ and $24 \mu \mathrm{m}$, indicating that PAH and hot dust exist towards this bubble, suggests that it could be a young HII region.

\section{Distance}

G35.6 exhibits a radio recombination line at $v_{\mathrm{LSR}} \sim$ $60 \mathrm{~km} \mathrm{~s}^{-1}$ (Lockman 1989), which, by applying the flat Galactic rotation curve of Fich et al. (1989) that assumes circular rotation around the Galactic center, gives the possible kinematic distances of $\sim 4.0$ or $\sim 9.8 \mathrm{kpc}$. This ambiguity arises because we are studying a region in the first Galactic quadrant, where a given velocity may be associated with two possible distances. Using HI data, we performed an absorption study towards the radio sources G35.6 (NVSS 185929+020334) and NVSS 185938+020012. Figure 2 shows the HI spectra towards both sources. The HI emission obtained over the source (the On position: a beam over the radio maximum of the source) is presented in red, in blue we present the average $\mathrm{HI}$ emission taken from four positions separated approximately by a beam from the source in the direction of the four Galactic cardinal points (the Off position), and the subtraction between them is presented in black, which has a $3 \sigma$ uncertainty of $\sim 10 \mathrm{~K}$. The figure shows that both sources have similar HI absorption features, suggesting that they are located at the same distance. The last absorption feature appears at $v \sim 61 \mathrm{~km} \mathrm{~s}^{-1}$, in coincidence with the G35.6 recombination line (Lockman 1989). Taking into account that the tangent point (at $v \sim 89.7 \mathrm{~km} \mathrm{~s}^{-1}$ ) does not exhibit any absorption, following Kolpak et al. (2003), we favour the near kinematic distance.

\section{Molecular analysis}

We analyze the whole ${ }^{13} \mathrm{CO} J=1-0$ data cube and find some interesting molecular structures between 47 and $60 \mathrm{~km} \mathrm{~s}^{-1}$. Figure 3 displays the integrated velocity channel maps of the ${ }^{13} \mathrm{CO} J=1-0$ emission every $\sim 1 \mathrm{~km} \mathrm{~s}^{-1}$, showing the kinematical and morphological structure of a molecular cloud probably related to the HII region G35.6. Between $\sim 48$ and $51 \mathrm{~km} \mathrm{~s}^{-1}$, a molecular structure appears delineating the PDR that extends to the south. No molecular gas is observed between 51 and $54 \mathrm{~km} \mathrm{~s}^{-1}$ (this velocity interval is not shown in Fig. 3). Finally, between 54 and $59 \mathrm{~km} \mathrm{~s}^{-1}$, several molecular clumps appear 

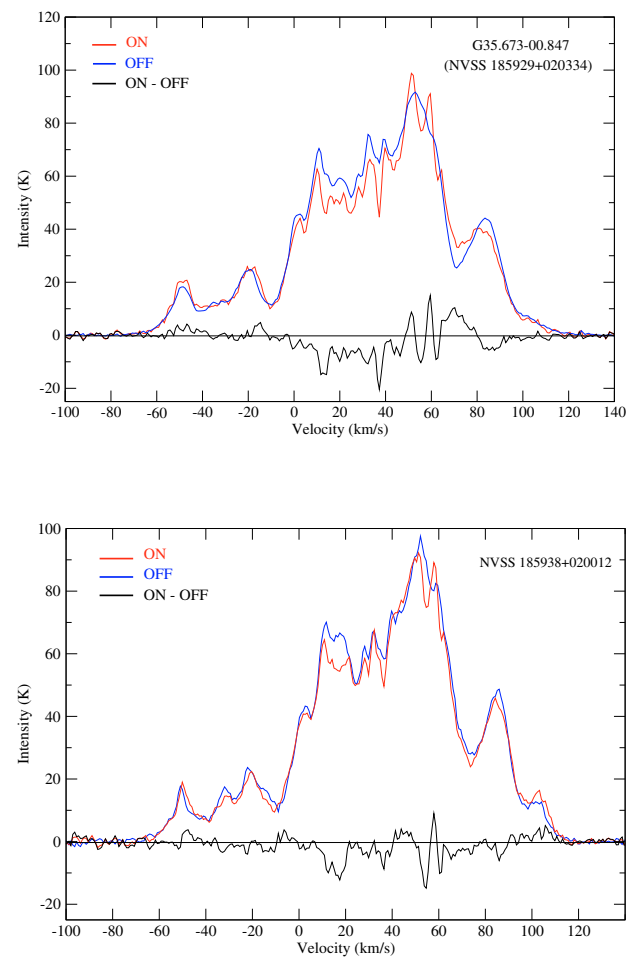

Fig. 2. Up: HI spectra obtained towards the source G35.6 (NVSS 185929+020334). Bottom: HI spectra obtained towards the source NVSS 185938+020012. The spectra obtained towards the sources (the On position) are presented in red, and in blue we present the averaged $\mathrm{HI}$ emission taken from four positions separated approximately by a beam from the source in the direction of the four Galactic cardinal points (the Off position), and the subtractions between them are presented in black. The $3 \sigma$ uncertainty in the subtraction is $\sim 10 \mathrm{~K}$.

distributed over the borders of G35.6 and the southern PDR, which may indicate that the collect and collapse process could be taking place in this region. As Deharveng et al. (2005) point out, the presence of a dense molecular shell surrounding the ionized gas of an HII region, or massive fragments regularly spaced along the ionization front, may be indicative of the collect and collapse mechanism. Figure 4 shows the ${ }^{13} \mathrm{CO} J=1-0$ emission integrated between 53 and $61 \mathrm{~km} \mathrm{~s}^{-1}$ (in green) over the $8 \mu \mathrm{m}$ emission (in red). The very good correspondence between the eastern HII region border, traced by the IR emission, and the molecular gas, strongly suggests that the observed molecular shell has been swept and shaped by the expansion of G35.6. The central velocity of the molecular gas is $\sim 57 \mathrm{~km} \mathrm{~s}^{-1}$, which infers a kinematic distance of either 3.7 or $10.1 \mathrm{kpc}$. According to the study presented in Sect. 4, we favour the nearest one. Taking into account that the ionized gas may be moving away from the molecular material, we use the central velocity of the molecular gas to adopt $3.7 \mathrm{kpc}$ as the distance of the whole complex.

To estimate the mass and density of the described molecular shell, we assume LTE, an excitation temperature of $20 \mathrm{~K}$, a distance of $3.7 \mathrm{kpc}$, and that the ${ }^{13} \mathrm{CO}$ emission is optically thin. From the standard LTE equations, we obtain a $\mathrm{N}\left({ }^{13} \mathrm{CO}\right)$ $\sim 3 \times 10^{16} \mathrm{~cm}^{-2}$, and using the relation $N\left(\mathrm{H}_{2}\right) / N\left({ }^{13} \mathrm{CO}\right) \sim 5 \times 10^{5}$ (e.g. Simon et al. 2001) we obtain a molecular mass and a density of $\sim 1.5 \times 10^{4} M_{\odot}$ and $\sim 1 \times 10^{4} \mathrm{~cm}^{-3}$, respectively. The integration was performed over all the observed positions within the $4.5 \mathrm{~K} \mathrm{~km} \mathrm{~s}^{-1}$ contour level shown in Fig. 4, following the shell geometry in the $55 \mathrm{~km} \mathrm{~s}^{-1}$ channel map in Fig. 3, i.e., the molecular condensation extending towards the southeast was not
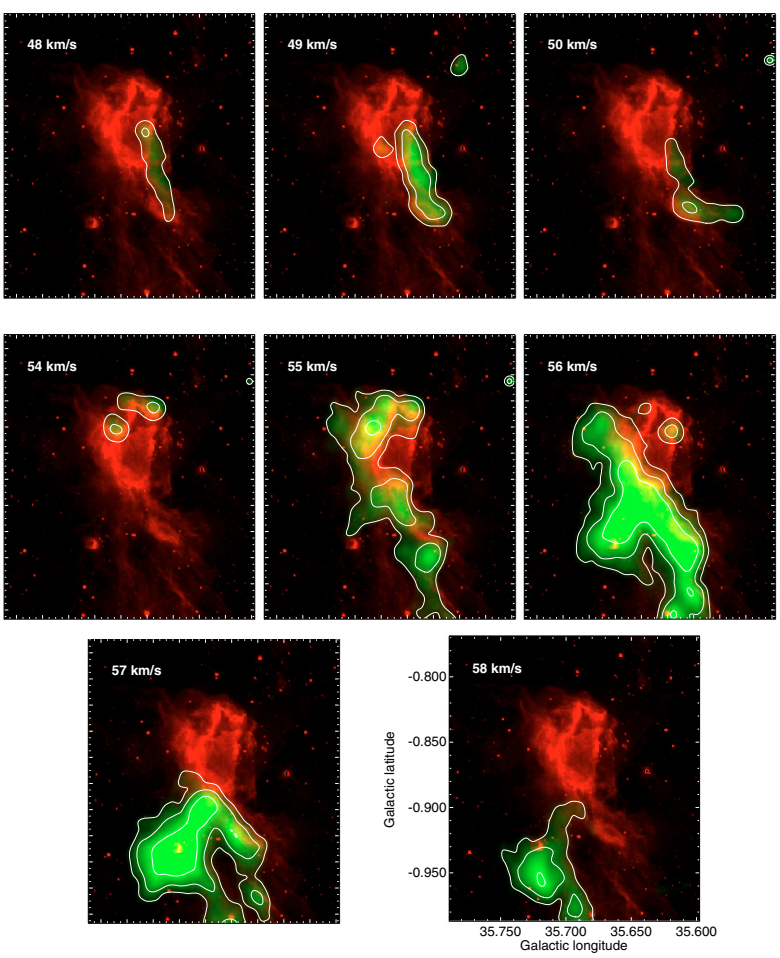

Fig. 3. Integrated velocity channel maps of the ${ }^{13} \mathrm{CO} J=1-0$ emission (in green) every $\sim 1 \mathrm{~km} \mathrm{~s}^{-1}$ in two velocity intervals: from $\sim 48$ to $51 \mathrm{~km} \mathrm{~s}^{-1}$ (shown in the first three panels) and from $\sim 54$ to $59 \mathrm{~km} \mathrm{~s}^{-1}$ (shown in the remaining panels). The contour levels of the ${ }^{13} \mathrm{CO} J=1-0$ emission are 1,2 and $4 \mathrm{~K} \mathrm{~km} \mathrm{~s}^{-1}$. Red is the $8 \mu \mathrm{m}$ emission.

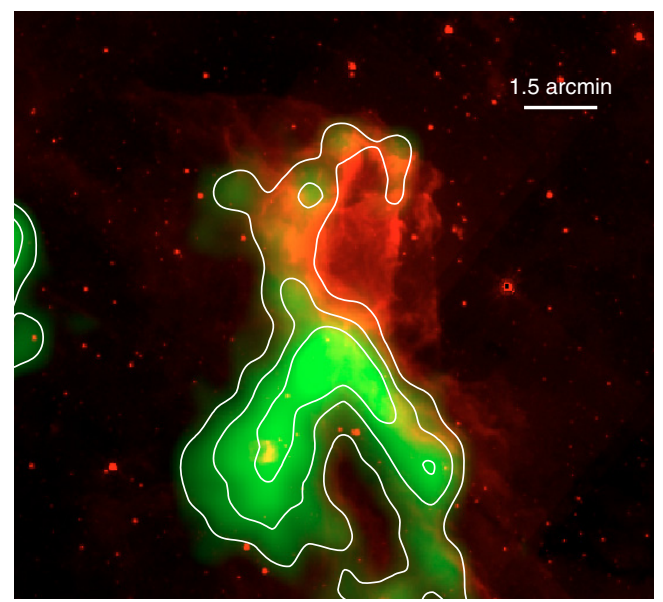

Fig. 4. ${ }^{13} \mathrm{CO} J=1-0$ emission (in green) integrated between 53 and $61 \mathrm{~km} \mathrm{~s}^{-1}$. The contour levels of the ${ }^{13} \mathrm{CO} J=1-0$ emission are 4.5, 8 and $12 \mathrm{~K} \mathrm{~km} \mathrm{~s}^{-1}$. Red is the $8 \mu \mathrm{m}$ emission.

considered. To calculate the volume of the molecular shell, we assume a length along the line of sight of $\sim 1^{\prime}(\sim 1.1 \mathrm{pc}$ at the distance of $3.7 \mathrm{kpc}$ ), which is approximately the average of the shell width seen in the plane of the sky (see the $55 \mathrm{~km} \mathrm{~s}^{-1}$ channel map in Fig. 3). On the other hand, we note that the little bubble described in Sect. 3, which is probably a young HII region, is likely to be embedded in this molecular condensation, which appears to be active in star formation. 
Table 1. Exciting star candidates in regions R1 and R2.

\begin{tabular}{|c|c|c|c|c|c|c|c|c|c|}
\hline$\#$ & 2MASS Designation & $J$ & $H$ & $K$ & $A_{v}$ & $M_{J}$ & $M_{H}$ & $M_{K}$ & O-type star \\
\hline & & & & & RI & & & & \\
\hline 1 & $\mathrm{~J} 18593297+0202530$ & 11.92 & 11.52 & 11.40 & 4.18 & -2.10 & -2.05 & -1.91 & - \\
\hline 2 & $\mathrm{~J} 18593204+0204495$ & 16.67 & 13.34 & 11.69 & 30.26 & -4.70 & -4.80 & -4.54 & yes \\
\hline 3 & $\mathrm{~J} 18592664+0204362$ & 13.90 & 10.54 & 8.86 & 30.61 & -7.58 & -7.66 & -7.41 & - \\
\hline 4 & $\mathrm{~J} 18593563+0203404$ & 15.05 & 12.25 & 10.88 & 25.44 & -4.97 & -5.05 & -4.81 & yes \\
\hline 5 & $\mathrm{~J} 18593189+0203454$ & 14.66 & 12.11 & 11.01 & 22.12 & -4.42 & -4.60 & -4.31 & yes \\
\hline 6 & $\mathrm{~J} 18592786+0203057$ & 14.14 & 11.88 & 10.92 & 19.58 & -4.23 & -4.39 & -4.11 & yes \\
\hline 7 & $\mathrm{~J} 18593076+0205225$ & 11.11 & 10.60 & 10.52 & 4.31 & -2.95 & -2.99 & -2.80 & - \\
\hline 8 & $\mathrm{~J} 18592979+0202149$ & 13.35 & 11.05 & 10.05 & 20.17 & -5.18 & -5.32 & -5.05 & - \\
\hline 9 & $\mathrm{~J} 18593057+0202486$ & 13.28 & 10.70 & 9.53 & 22.81 & -6.00 & -6.13 & -5.87 & - \\
\hline 10 & $\mathrm{~J} 18592697+0202073$ & 12.66 & 10.11 & 8.89 & 23.09 & -6.69 & -6.77 & -6.53 & - \\
\hline 11 & $\mathrm{~J} 18593472+0204518$ & 16.34 & 13.32 & 11.88 & 27.07 & -4.13 & -4.26 & -3.99 & yes \\
\hline 12 & $\mathrm{~J} 18592785+0203304$ & 11.69 & 10.30 & 9.56 & 13.83 & -5.05 & -4.96 & -4.83 & yes \\
\hline 13 & $\mathrm{~J} 18593016+0204431$ & 13.84 & 10.65 & 8.90 & 30.39 & -7.57 & -7.51 & -7.35 & - \\
\hline 14 & $\mathrm{~J} 18593041+0202363$ & 14.38 & 12.47 & 11.64 & 16.95 & -3.24 & -3.34 & -3.10 & yes \\
\hline 15 & $\mathrm{~J} 18592897+0202470$ & 11.54 & 9.09 & 7.87 & 22.60 & -7.67 & -7.71 & -7.50 & - \\
\hline & & & & & $R 2$ & & & & \\
\hline 16 & $\mathrm{~J} 18593584+0200579$ & 14.35 & 12.37 & 11.51 & 17.50 & -3.43 & -3.53 & -3.29 & yes \\
\hline 17 & $\mathrm{~J} 18593743+0200411$ & 14.07 & 11.69 & 10.58 & 21.40 & -4.81 & -4.90 & -4.66 & yes \\
\hline 18 & $\mathrm{~J} 18593556+0200488$ & 13.81 & 11.97 & 11.25 & 15.70 & -3.46 & -3.62 & -3.35 & yes \\
\hline 19 & $\mathrm{~J} 18594015+0200255$ & 15.76 & 12.94 & 11.67 & 24.73 & -4.06 & -4.23 & -3.94 & yes \\
\hline 20 & $\mathrm{~J} 18593887+0200288$ & 15.20 & 12.82 & 11.71 & 21.40 & -3.68 & -3.77 & -3.53 & yes \\
\hline 21 & $\mathrm{~J} 18593725+0201066$ & 15.22 & 13.21 & 12.25 & 18.47 & -2.83 & -2.86 & -2.66 & - \\
\hline
\end{tabular}

Notes. The sources number correspond to the numeration in Fig. 5.

\section{Exciting stars}

No exciting star of the HII region G35.6 can be found in the literature. In this work, we provide indirect evidence of the location and properties of the exciting star(s) of the region.

The first piece of information is given by the radio continuum emission of G35.6, which allow us to derive the expected spectral type of the exciting star. The number of UV ionizing photons needed to keep an HII region ionized is given by $N_{\mathrm{uv}}=0.76 \times 10^{47} T_{4}^{-0.45} v_{\mathrm{GHz}}^{0.1} S_{v} D_{\mathrm{kpc}}^{2}$ (Chaisson 1976), where $T_{4}$ is the electron temperature in units of $10^{4} \mathrm{~K}, D_{\mathrm{kpc}}$ the distance in $\mathrm{kpc}, v_{\mathrm{GHz}}$ the frequency in $\mathrm{GHz}$, and $S_{v}$ the measured total flux density in Jy. Assuming an electron temperature of $T=10^{4} \mathrm{~K}$, a distance of $3.7 \mathrm{kpc}$, and using a total flux density of $0.86 \mathrm{Jy}$ at $2.7 \mathrm{GHz}$ for G35.6 (Reich et al. 1984) and a total flux density of $0.053 \mathrm{Jy}$ at $1.4 \mathrm{GHz}$ for NVSS $185938+020012$ (Condon et al. 1998), the total amount of ionizing photons needed to keep these sources ionized turns out to be about $N_{\mathrm{uv}}=1.0 \times 10^{48} \mathrm{ph} \mathrm{s}^{-1}$ and $N_{\text {uv }}=0.6 \times 10^{47} \mathrm{ph} \mathrm{s}^{-1}$, respectively. It is well established that part of the UV radiation can be dissipated in heating the dust. Inoue (2001) and Inoue et al. (2001), indeed demonstrated that typically only half of the Lyman continuum photons from the central source in a Galactic HII region ionizes neutral hydrogen, the remainder being absorbed by dust grains within the ionized region. We take this into account, consider errors of about ten percent in both the distance and the radio continuum flux at $2.7 \mathrm{GHz}$, and base on the ionizing fluxes for massive stars given by Martins et al. (2005) to estimate the spectral type of the ionizing star of G35.6 to be between O7.5V and O9V.

In addition we perform a photometric study of the infrared point sources in the region based on the GLIMPSE I Spring'07 and the 2MASS All-Sky Point Source Catalogs. Only sources with detections in the four Spitzer-IRAC bands and the three 2MASS bands were considered. We find 30 and 8 sources towards both the HII region G35.6 (R1 in Fig. 5) and the source NVSS $185938+020012$ (R2 in Fig. 5), respectively. To examine the evolutionary stage of the infrared point sources, we analyze

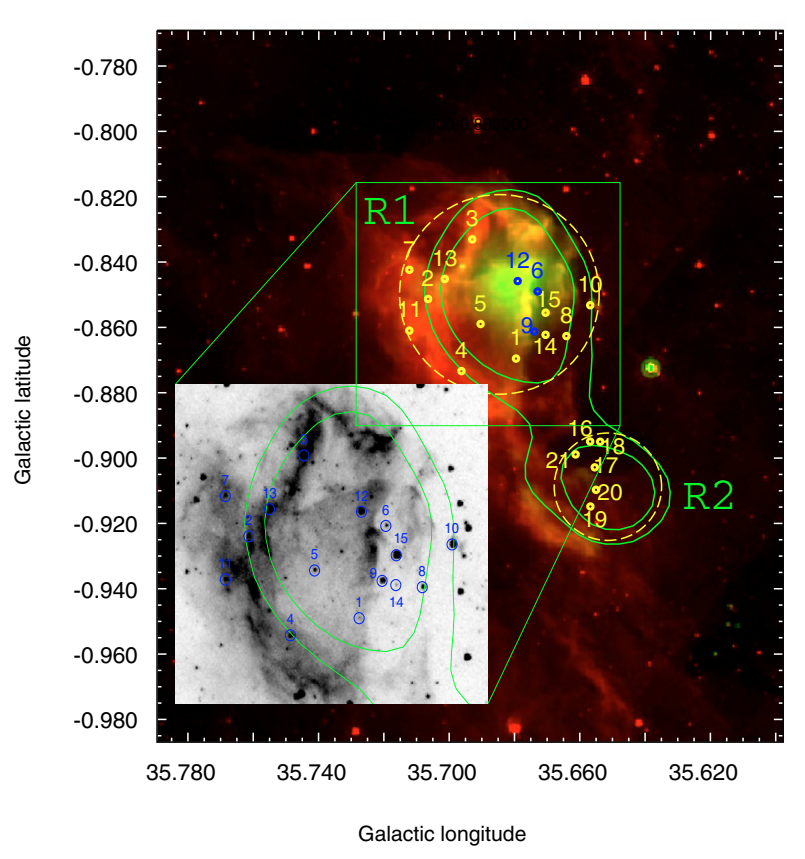

Fig. 5. Spitzer-IRAC two-color image $(8 \mu \mathrm{m}=$ red and $24 \mu \mathrm{m}=$ green $)$. The green contours represent the radio continuum emission at $20 \mathrm{~cm}$. The crosses show the location of the main sequence star candidates in R1 and R2 (dashed circles).

their location in a color-color IRAC diagram. Following Allen et al. (2004) color criteria, we found 15 and 6 sources in R1 and $\mathrm{R} 2$, respectively, which can be classified as main sequence stars (Class III). Table 1 presents these sources with their 2MASS designation (Col. 2), apparent $J H K$ magnitudes (Cols. 3-5), estimated extinctions (Col. 6), calculated absolute JHK magnitudes (Cols. 7-9), and indication whether their derived spectral type coincides with an O-type in Col. 10. The errors in the estimated 
extinctions and the calculated absolute $J H K$ magnitudes are below $20 \%$ and $30 \%$, respectively. The sources are labeled according to Fig. 5, which displays their location in a two-color image, where the $8 \mu \mathrm{m}$ and $24 \mu \mathrm{m}$ emissions are displayed in red and green, respectively. The green contours delineate the radio continuum emission at $20 \mathrm{~cm}$. To search for O-type stars (probably responsible for ionizing the surrounding gas), we use the $J$, $H$, and $K$ apparent magnitudes obtained from the 2MASS Point Source Catalog to derive the absolute $J H K$ magnitudes. To perform that, we assume a distance of about $3.7 \mathrm{kpc}$ and obtain the extinction for each source from the $(J-H)$ and $(H-K)$ colors. We assume the interstellar reddening law of Rieke \& Lebofsky (1985) $\left(A_{J} / A_{V}=0.282, A_{H} / A_{V}=0.175\right.$, and $\left.A_{K} / A_{V}=0.112\right)$ and the intrinsic colors $(J-H)_{0}$ and $(H-K)_{0}$ obtained from Martins \& Plez (2006). By comparing the derived absolute magnitudes with those tabulated by Martins \& Plez (2006), we find that seven sources in region $1, \# 2, \# 4, \# 5, \# 6$, \#11, \#12, and \#14, and five sources in region 2, \#16, \#17, \#18, \#19, and \#20, have absolute $J H K$ magnitudes that agree with those of an O-type star (see Table 1).

Finally, taking into account that the exciting star candidates are expected to be in a PAH hole, we exclude sources \#2, \#4, and \#11 as the responsible of generate G35.6. The rest of the exciting star candidates, sources \#5, \#6, \#12, and \#14, are located in projection within the radio continuum and $24 \mu \mathrm{m}$ emissions; among them, sources \#6 and \#12 are located close to the maximum of the $24 \mu \mathrm{m}$ emission as expected for an exciting star. On the other hand, as can be seen in Fig. 5, source \#6 is located in a hole of the $5.8 \mu \mathrm{m}$ emission (see zoom of the region in the figure). It is well known that the exciting star(s) of an HII region generates a cavity of dust and gas because of the action of the radiation pressure on the dust grains (Gail \& Sedlmayr 1979), which suggests that source \#6 is the more likely exciting-star candidate of G35.6. On the other hand, using the same assumptions as for R1, we found that based on the radio continuum flux at $1.4 \mathrm{GHz}$ in $\mathrm{R} 2$, the exciting stars of NVSS $185938+020012$ would be later than an O9.5V star. The later spectral type stars found in R2 could be the sources \#16 and \#18.

It would be very useful to have UBV fluxes to perform more accurate photometry and identify beyond doubt the exciting stars, although these fluxes are very difficult to obtain because of the interstellar absorption towards this region of the Galaxy.

\section{Star formation}

In Sect. 5, we show that the HII region G35.6 is evolving and affecting a molecular cloud, presenting an excellent scenario for probing triggered star formation. In this section, we look for young stellar objects (YSOs) around G35.6. YSOs are generally classified according to their evolutionary stage: class I are the youngest sources embedded in dense envelopes of gas and dust, and class II are sources whose emission originates mainly in the accretion disk around the central protostar. In both cases, a YSO exhibits an infrared excess that cannot be attributed to the scattering and absorption of the ISM along the line of sight. In contrast, this infrared excess is mainly caused by the envelope and/or the disk of dust around the central protostar. In other words, YSOs are intrinsically red sources.

Robitaille et al. (2008) defined a color criterion to identify intrinsically red sources using data from the Spitzer-IRAC bands. Intrinsically red sources satisfy the condition $m_{4.5}-m_{8.0} \geq 1$, where $m_{4.5}$ and $m_{8.0}$ are the magnitudes in the 4.5 and $8.0 \mu \mathrm{m}$ bands, respectively. On the other hand, an externally reddened

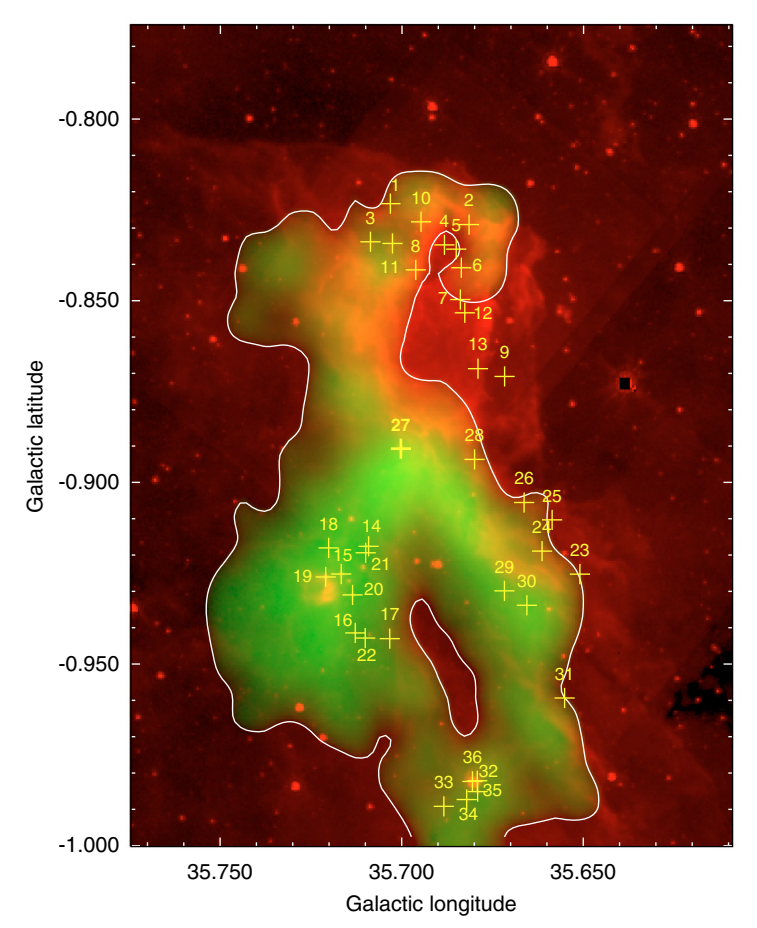

Fig. 6. Two-color image: the ${ }^{13} \mathrm{CO}$ emission integrated between 53 and $61 \mathrm{~km} \mathrm{~s}^{-1}$ is presented in green, and the $8 \mu \mathrm{m}$ emission, in red. For sharper contrast, the ${ }^{13} \mathrm{CO}$ emission scale is displayed in square root and bordered by a white contour. The yellow crosses indicate the position of the intrinsically red sources, i.e., sources satisfying the condition $m_{4.5}-$ $m_{8.0}+\varepsilon \geq 1$. We labeled the sources that appear to be related to the molecular gas around G35.6.

source is a source that is not intrinsically red (such as mainsequence stars) but appears red because of interstellar effects. They satisfy the condition $m_{4.5}-m_{8.0}<1$ and their spectral energy distributions (SEDs) are well fitted by stellar photosphere models with interstellar extinction. To consider the errors in the magnitudes, we use a color criterion to select intrinsically red sources given by $m_{4.5}-m_{8.0}+\varepsilon \geq 1$, where $\varepsilon=\sqrt{\left(\Delta_{4.5}\right)^{2}+\left(\Delta_{8.0}\right)^{2}}$ and $\Delta_{4.5}$ and $\Delta_{8.0}$ are the errors in the 4.5 and $8.0 \mu \mathrm{m}$ bands, respectively. In Fig. 6, we show the distribution of the sources extracted from the GLIMPSE catalog around G35.6 that satisfy the previous criterion (we considered only sources with detections in both of the 4.5 and $8.0 \mu \mathrm{m}$ bands). The intrinsically red sources are distributed into four groups. The first group (group 1) is found towards the north and includes the sources from 1 to 13 . A second group (group 2) of sources is located over the southeastern molecular structure. This portion of the molecular cloud is far from G35.6 and probably not being perturbed by the HII region. Sources 23, 24, 25, 26, 29, and 30 form group 3, which appears in the molecular gas to be likely associated with the border of the radio continuum source NVSS 185938+020012. We then identify a fourth group (group 4) towards the southern portion of the molecular cloud, far from the HII regions. Finally, sources 27, 28, and 31 are not part of any group. Sources 27 and 28, taking into account their position, could be related to the G35.6 southern border. In Table 2, we report the fluxes of the intrinsically red sources in the 2MASS and Spitzer-IRAC bands, specifying the GLIMPSE designation (Col. 2) and the 2MASS photometric quality (Col. 3). In the case of sources $8,15,19,20$, 21,27 , and 30 , we derived their fluxes at $24 \mu \mathrm{m}$ from the MIPS image and present these in Col. 11 of the table. 
A\&A 525, A132 (2011)

Table 2. Near- and mid-IR fluxes of the sources satisfying the condition $m_{4.5}-m_{8.0}+\varepsilon \geq 1$ around G35.6.

\begin{tabular}{|c|c|c|c|c|c|c|c|c|c|c|}
\hline Source & GLIMPSE Desig. & 2MASS Qual. & $\begin{array}{c}J \\
(\mathrm{mag})\end{array}$ & $\begin{array}{c}H \\
(\mathrm{mag})\end{array}$ & $\begin{array}{c}K_{S} \\
(\mathrm{mag})\end{array}$ & $\begin{array}{c}3.6 \mu \mathrm{m} \\
(\mathrm{mag})\end{array}$ & $\begin{array}{c}4.5 \mu \mathrm{m} \\
(\mathrm{mag})\end{array}$ & $\begin{array}{c}5.8 \mu \mathrm{m} \\
(\mathrm{mag})\end{array}$ & $\begin{array}{c}8.0 \mu \mathrm{m} \\
(\mathrm{mag})\end{array}$ & $\begin{array}{c}24 \mu \mathrm{m} \\
(\mathrm{Jy})\end{array}$ \\
\hline YSO 1 & G035.7031-00.8232 & BAA & 16.513 & 14.154 & 12.967 & 12.086 & 11.603 & 11.258 & 10.285 & \\
\hline YSO 2 & G035.6814-00.8290 & AAA & 15.830 & 13.625 & 12.495 & 11.284 & 10.692 & 10.049 & 9.182 & \\
\hline YSO 3 & G035.7086-00.8337 & BAA & 16.641 & 13.712 & 12.405 & 11.423 & 11.343 & 10.609 & 10.009 & \\
\hline YSO 4 & G035.6882-00.8346 & UUA & 18.264 & 15.119 & 14.434 & 12.445 & 11.992 & 11.545 & 10.877 & \\
\hline YSO 5 & G035.6850-00.8358 & UAA & 17.304 & 15.046 & 13.740 & 12.101 & 11.557 & 11.057 & 10.513 & \\
\hline YSO 6 & G035.6836-00.8409 & UAE & 18.038 & 15.025 & 13.485 & 11.546 & 11.054 & 10.487 & 9.958 & \\
\hline YSO 7 & G035.6838-00.8496 & UAA & 17.579 & 15.185 & 14.077 & 12.942 & 12.344 & 12.307 & 11.169 & \\
\hline YSO 8 & G035.6961-00.8415 & N/A & & & & 13.176 & 11.297 & 9.674 & 8.558 & 0.14 \\
\hline YSO 9 & G035.6716-00.8708 & AAA & 15.493 & 13.459 & 12.624 & 11.955 & 11.845 & 11.431 & 10.973 & \\
\hline YSO 10 & G035.6947-00.8282 & N/A & & & & 13.616 & 13.762 & & 9.994 & \\
\hline YSO 11 & G035.7025-00.8342 & N/A & & & & 14.065 & 13.908 & & 9.828 & \\
\hline YSO 12 & G035.6826-00.8533 & N/A & & & & 14.114 & 13.431 & & 10.776 & \\
\hline YSO 13 & G035.6790-00.8687 & N/A & & & & 13.970 & 13.690 & & 10.811 & \\
\hline YSO 14 & G035.7094-00.9176 & N/A & & & & 13.583 & 13.145 & 11.983 & 11.299 & \\
\hline YSO 15 & G035.7166-00.9252 & AAA & 15.776 & 13.607 & 12.112 & 10.110 & 9.568 & 8.992 & 8.213 & 0.05 \\
\hline YSO 16 & G035.7127-00.9414 & AAA & 15.713 & 14.113 & 13.148 & 11.797 & 11.196 & 10.718 & 10.142 & \\
\hline YSO 17 & G035.7032-00.9430 & N/A & & & & 13.242 & 12.489 & 11.977 & 11.313 & \\
\hline YSO 18 & G035.7201-00.9180 & N/A & & & & 14.267 & 15.532 & 12.876 & 11.596 & \\
\hline YSO 19 & G035.7209-00.9260 & UAA & 18.218 & 14.992 & 13.379 & 10.983 & 9.856 & 9.109 & 8.363 & 0.16 \\
\hline YSO 20 & G035.7135-00.9309 & UUA & 18.257 & 16.988 & 13.519 & 11.184 & 10.301 & 9.740 & 9.235 & 0.05 \\
\hline YSO 21 & G035.7099-00.9193 & N/A & & & & & 13.787 & 12.354 & 11.781 & 0.03 \\
\hline YSO 22 & G035.7103-00.9428 & UAA & 16.441 & 14.637 & 13.419 & 12.754 & 12.716 & 11.904 & 11.944 & \\
\hline YSO 23 & G035.6509-00.9252 & $A$ & 15.825 & 13.779 & 12.688 & 12.018 & 11.926 & 11.673 & 10.935 & \\
\hline YSO 24 & G035.6613-00.9189 & UAA & 17.288 & 14.442 & 13.061 & 12.238 & 11.995 & 11.687 & 11.049 & \\
\hline YSO 25 & G035.6585-00.9103 & UAA & 18.328 & 15.205 & 13.736 & 12.791 & 12.505 & 12.328 & 11.523 & \\
\hline YSO 26 & G035.6663-00.9055 & 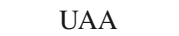 & 17.509 & 13.978 & 12.464 & 11.240 & 11.208 & 10.885 & 10.344 & \\
\hline YSO 27 & G035.7002-00.8907 & UUA & 14.254 & 12.706 & 13.451 & 10.951 & 9.755 & 8.856 & 8.321 & 0.14 \\
\hline YSO 28 & G035.6799-00.8936 & UAA & 17.204 & 15.356 & 14.118 & 13.152 & 12.933 & & 10.466 & \\
\hline YSO 29 & G035.6717-00.9298 & & & & & 13.379 & 12.823 & & 11.280 & \\
\hline YSO 30 & G035.6655-00.9338 & N/A & & & & 14.952 & 13.166 & 11.493 & 11.337 & 0.04 \\
\hline YSO 31 & G035.6551-00.9593 & AAA & 16.315 & 14.103 & 13.002 & 12.365 & 12.317 & 12.050 & 11.254 & \\
\hline YSO 32 & G035.6791-00.9821 & UAA & 15.364 & 14.161 & 12.039 & 10.210 & 9.916 & 9.250 & 8.524 & \\
\hline YSO 33 & G035.6883-00.9891 & BAA & 16.607 & 14.877 & 14.225 & 13.284 & 13.150 & & 11.873 & \\
\hline YSO 34 & G035.6820-00.9873 & UAA & 17.289 & 14.683 & 13.584 & 11.714 & 11.025 & 10.649 & 10.107 & \\
\hline YSO 35 & G035.6790-00.9851 & UUB & 18.284 & 15.686 & 14.581 & 11.914 & 10.942 & 10.260 & 9.520 & \\
\hline YSO 36 & G035.6804-00.9823 & UAA & 14.186 & 11.459 & 9.535 & 7.425 & 6.711 & 5.985 & 4.827 & \\
\hline
\end{tabular}

Notes. The numbers of YSO candidates correspond to the numeration in Fig. 6. 2MASS Qual.: A and B are the best photometric qualities, with a $S N R \leq 10$ and $\leq 7$, respectively. E means that the source magnitude is questionable. And $\mathrm{U}$ means that the magnitude value is an upper limit.

We identified the intrinsically red sources located in the molecular gas around the HII region G35.6. However, according to Robitaille et al. (2008) intrinsically red sources may include YSOs, planetary nebulae (PNe), galaxies, AGNs, and AGB stars. We had to apply an additional constraint to these sources to discern their real nature. Regarding extragalactic sources, Robitaille et al. (2008) pointed out that at most $0.4 \%$ of the intrinsically red sources selected by the color criterion $m_{4.5}-m_{8.0}>1$ are galaxies and AGNs. Hence, there is a low probability of finding an extragalactic source in our small sample of red sources. To identify AGB stars, we searched in catalogues of these stars. In the region analyzed in this work, no AGB star was catalogued. To search for YSOs and PNe candidates, we constructed a color-color (CC) diagram [5.8]-[8.0] versus [3.6]-[4.5] with the sources from Table 2 that have flux detections in the four Spitzer-IRAC bands. We used the photometric criteria of Allen et al. (2004) to identify class I and II YSOs (Fig. 7). From their positions in this color-color diagram, we found that only source 22 cannot be classified as either a class I or II YSO. Source 30 falls outside the class I region but if we take into account the errors in the fluxes we should consider it as a YSO candidate. On the other hand, sources 7 and 18 are found in the CC diagram close to the locations of PNe, which typically have [5.8]-[8.0] > 1.4 (Cohen et al. 2007). However, we will consider them as YSO candidates too.

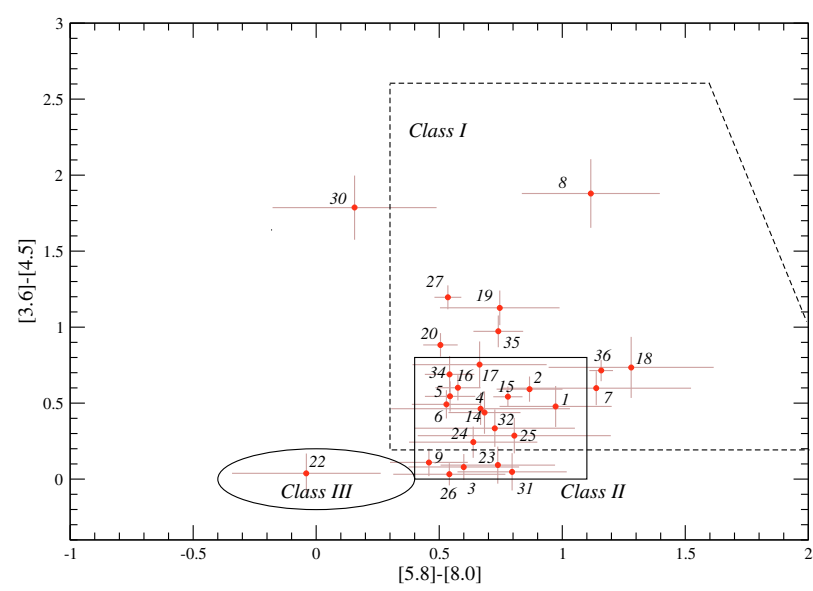

Fig. 7. Color-color diagram [5.8]-[8.0] versus [3.6]-[4.5] for sources of Table 2 with detections in the four Spitzer-IRAC bands. The regions indicate the stellar evolutionary stage based on the photometric criteria of Allen et al. (2004).

Finally, we fitted the spectral energy distribution (SED) of the sources from which we obtained fluxes at $24 \mu \mathrm{m}$ from the MIPS image using the tool developed by Robitaille et al. (2007) available online ${ }^{3}$. We assume an interstellar absorption between

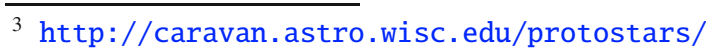


Table 3. Parameters derived from the SED fitting of sources from which we obtained fluxes at $24 \mu \mathrm{m}$ from the MIPS image.

\begin{tabular}{ccccccccc}
\hline \hline Source & $\chi_{\text {YSO }}^{2} / N$ & $\chi_{\star}^{2} / N$ & $n$ & $\begin{array}{c}M_{\star} \\
\left(M_{\odot}\right)\end{array}$ & $\begin{array}{c}M_{\text {disk }} \\
\left(M_{\odot}\right)\end{array}$ & $\begin{array}{c}M_{\text {env }} \\
\left(M_{\odot}\right)\end{array}$ & $\begin{array}{c}\dot{M}_{\text {env }} \\
\left(M_{\odot} / \text { yr }\right)\end{array}$ & Stage \\
\hline YSO 8 & 1 & 520 & 12 & $1-4$ & $1 \times 10^{-3}-2 \times 10^{-1}$ & $5 \times 10^{-8}-3 \times 10^{1}$ & $0-3 \times 10^{-4}$ & I \\
YSO 15 & 0.4 & 50 & 109 & $3-5$ & $1 \times 10^{-5}-1 \times 10^{-1}$ & $6 \times 10^{-9}-2$ & $0-2 \times 10^{-7}$ & II \\
YSO 19 & 1 & $>9999$ & 7 & 4 & $3 \times 10^{-3}-5 \times 10^{-2}$ & $2 \times 10^{-4}-8 \times 10^{-2}$ & $0-9 \times 10^{-6}$ & I, II \\
YSO 20 & 28 & $>9999$ & 1 & 5 & $3 \times 10^{-4}$ & $4 \times 10^{-6}$ & 0 & II \\
YSO 21 & 0.1 & 505 & 72 & $0.2-5$ & $6 \times 10^{-4}-3 \times 10^{-1}$ & $9 \times 10^{-3}-2 \times 10^{2}$ & $2 \times 10^{-6}-9 \times 10^{-4}$ & I \\
YSO 30 & 1.5 & 580 & 12 & $2-13$ & $2 \times 10^{-3}-5 \times 10^{-1}$ & $2-5 \times 10^{2}$ & $5 \times 10^{-5}-6 \times 10^{-3}$ & I \\
YSO 27 & 1.3 & $>9999$ & 2 & $8-18$ & $0-4 \times 10^{-2}$ & $7 \times 10^{1}-2 \times 10^{2}$ & $8 \times 10^{-5}-2 \times 10^{-3}$ & I \\
\hline
\end{tabular}

12 and 22 mag. These values were obtained from the 2MASS $J-H$ versus $H$ - $K s$ color-color diagram (not presented here) constructed with the sources with the highest photometric quality (AAA) within a circle of $8^{\prime}$ in radius centered on G35.6. The lower value is compatible with the expected extinction towards star-forming regions, which, according to Neckel \& Klare (1980), is generally greater than $10 \mathrm{mag}$. The upper value agrees with the visual absorption of $A_{v} \sim 20 \mathrm{mag}$ obtained from $A_{v}=5 \times 10^{-22} N(\mathrm{H})$ (Bohlin et al. 1978), where $N(\mathrm{H})=$ $N(\mathrm{HI})+2 N\left(\mathrm{H}_{2}\right)$ is the line-of-sight hydrogen column density towards this region, which is about $4 \times 10^{22} \mathrm{~cm}^{-2}$. This value was obtained from both the HI column density derived from the VGPS HI data and the $\mathrm{H}_{2}$ column density inferred by the ${ }^{13} \mathrm{CO}$ $J=1-0$ data.

The SED good fitting models are selected according to the condition $\chi^{2}-\chi_{\text {YSO }}^{2}<2 N$, where $\chi_{\text {YSO }}^{2}$ is the $\chi^{2}$ of the YSO best-fit model and $N$ is the number of input data fluxes (fluxes specified as upper limit do not contribute to $N$ ). Hereafter, we refer to models satisfying the above equation as "selected models". The fitting tool also fits the data to a stellar photosphere and defines the parameter $\chi_{\star}^{2}$ to evaluate the goodness of the fitting. By comparing $\chi_{\text {YSO }}^{2}$ with $\chi_{\star}^{2}$ we can confirm which sources are YSOs and which sources may be stars externally reddened by the ISM. The SED fitting allows us to establish the evolutionary stage of the YSO candidates by considering the physical parameters of the sources: the central source mass $M_{\star}$, the disk mass $M_{\text {disk }}$, the envelope mass $M_{\text {env }}$, and the envelope accretion rate $\dot{M}_{\text {env }}$. According to Robitaille et al. (2006): stage I YSOs are those that have $\dot{M}_{\text {env }} / M_{\star}>10^{-6} \mathrm{yr}^{-1}$, i.e., protostars with large accretion envelopes; stage II are those with $M_{\text {disk }} / M_{\star}>10^{-6}$ and $\dot{M}_{\text {env }} / M_{\star}<10^{-6} \mathrm{yr}^{-1}$, i.e., young objects with prominent disks; and stage III are those with $M_{\text {disk }} / M_{\star}<10^{-6}$ and $\dot{M}_{\text {env }} / M_{\star}<10^{-6} \mathrm{yr}^{-1}$, i.e., evolved sources whose flux is dominated by that of the central source.

In Table 3, we report the main results of the fitting output for the YSO candidates from which we obtained fluxes at $24 \mu \mathrm{m}$ from the MIPS image. In Cols. 2 and 3, we report the $\chi^{2}$ per data point of the YSO and stellar photosphere best-fit model, respectively, and in Col. 4 the number of models satisfying the $\chi^{2}$ equation. The remaining columns report the physical parameters of the source, specifying the range of values of the selected models, i.e., the central source mass, disk mass, envelope mass, and envelope accretion rate, respectively. Following the criteria of Robitaille et al. (2006), the last column indicates the evolutionary stage inferred by inspecting the selected models. Figure 8 shows the SED of these sources. From this analysis, we can appreciate that for source 15 the flux at longer wavelengths originates mainly in the disk, indicating that it is a class II YSO, in coincidence with its position in the Spitzer-IRAC CC diagram. The SED for source 20 also shows the characteristics of a class II
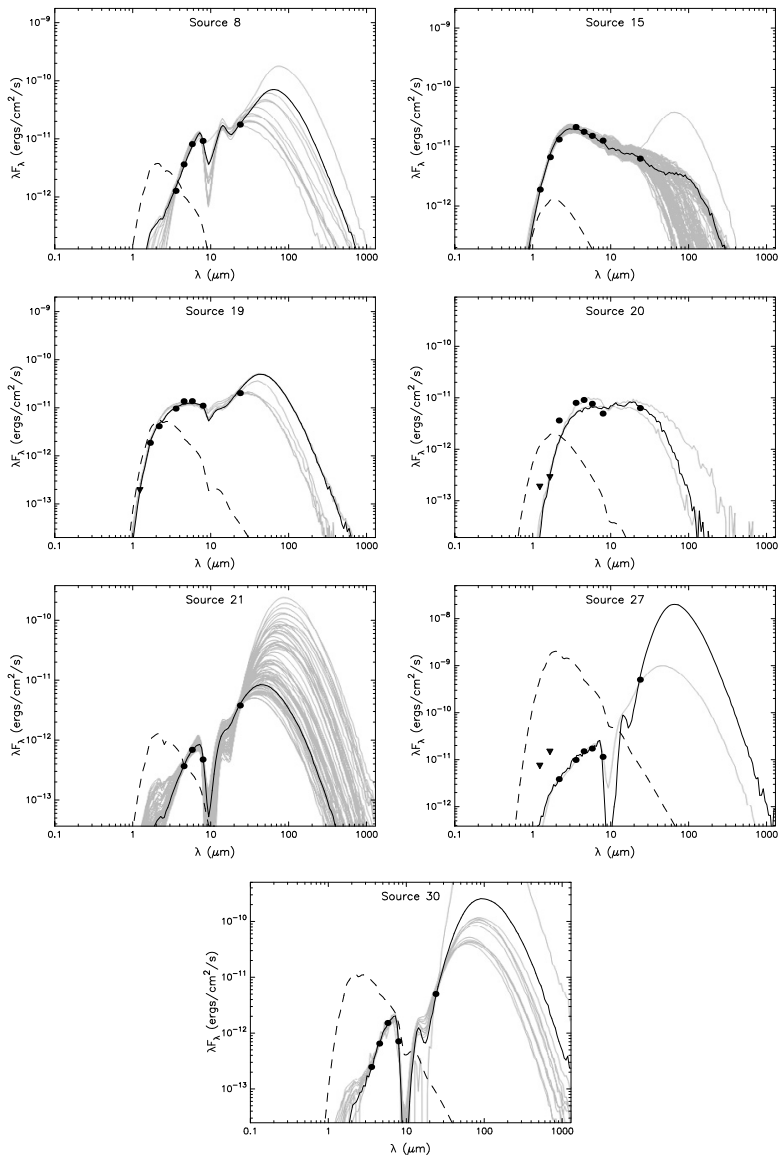

Fig. 8. SED of sources from which we obtained fluxes at $24 \mu \mathrm{m}$ from the MIPS image. The sources are numbered according to Table 3 and Figs. 6 and 7. In each panel, black line shows the best fit, and the gray lines show subsequent good fits. The dashed line shows the stellar photosphere corresponding to the central source of the best-fit model, as it would look in the absence of circumstellar dust. The points are the input fluxes.

YSO. In the case of source 19, the selected models indicate that this source could be stage I and II. For sources 8, 21, 27, and 30, the selected models are those of stage I and the SED shows that the flux at longer wavelengths is dominated by the envelope flux. These sources, together with source 19, are located in the region of a class I YSO in the CC diagram (except for source 21, which lacks flux at $3.6 \mu \mathrm{m}$ ), confirming their youth.

From Fig. 7 and the SED analysis, we can confirm the presence of YSOs around G35.6. Thus, we conclude that the region is indeed active in star formation and suggest that the birth of 
some YSOs, mainly those belonging to group 1 and sources 27 and 28 may have been triggered by the expansion of the HII region G35.6. Most of the remaining intrinsically red sources belonging to groups 2, 3, and 4 may also be YSOs but their position far from the HII region does not allow us to confirm that their formation was triggered by G35.6.

\section{Collect and collapse scenario}

To determine whether the collect and collapse mechanism is responsible for the star formation taking place in the periphery of the HII region G35.6, we estimate and compare the age of the HII region and the fragmentation time predicted by the theoretical models of Whitworth et al. (1994a,b).

Using a simple model described by Dyson \& Williams (1980), we calculate the age of the HII region at a given radius $R$ as

$t(R)=\frac{4 R_{\mathrm{S}}}{7 c_{\mathrm{S}}}\left[\left(\frac{R}{R_{\mathrm{S}}}\right)^{7 / 4}-1\right]$,

where $c_{\mathrm{S}}$ is the sound velocity in the ionized gas $\left(c_{\mathrm{S}}=10 \mathrm{~km} \mathrm{~s}^{-1}\right)$ and $R_{\mathrm{S}}$ is the radius of the Strömgren sphere given by $R_{\mathrm{S}}=$ $\left(3 N_{\mathrm{uv}} / 4 \pi n_{0}^{2} \alpha_{B}\right)^{1 / 3}$, where $\alpha_{B}=2.6 \times 10^{-13} \mathrm{~cm}^{3} \mathrm{~s}^{-1}$ is the hydrogen recombination coefficient to all levels above the ground level, $N_{\mathrm{uv}}$ is the total number of ionizing photons per unit of time emitted by the star(s), and $n_{0}$ is the original ambient density.

Taking into account the results of Sect. 6 , we consider a Lyman continuum photon flux of $1.0 \times 10^{48} \mathrm{ph} \mathrm{s}^{-1}$. Adopting a radius of $\sim 1.5^{\prime}$ for the HII region, a distance of $3.7 \mathrm{kpc}$, and an original ambient density of $\sim(1 \pm 0.5) \times 10^{3} \mathrm{~cm}^{-3}$, we derive a dynamical age of between 0.18 and $0.35 \mathrm{Myr}$ for G35.6. To coarsely estimate the original ambient density (assuming an error of $50 \%$ ), we distribute the above calculated mass of the molecular shell, $\sim 10^{4} M_{\odot}$, over an ellipsoid of revolution with semiaxes of 3 and $7 \mathrm{pc}$ that encloses the molecular and ionized gas.

As analyzed in Sect. 5, the morphology of the molecular gas that encircles the HII region suggests that the expansion of G35.6 collects the gas at its periphery. Finally, we ask whether the fragmentation of the collected layer of material can occur in the region. To answer this, we estimate when the fragmentation of the collected layer should occur according to Whitworth's models. Assuming a turbulent velocity in the collected layer $a_{\mathrm{s}}$ ranging between 0.2 and $0.6 \mathrm{~km} \mathrm{~s}^{-1}$ (Whitworth et al. 1994b), a Lyman continuum photon flux of $1.0 \times 10^{48} \mathrm{ph} \mathrm{s}^{-1}$, and the previously estimated original ambient density of $\sim(1 \pm 0.5) \times 10^{3} \mathrm{~cm}^{-3}$, we find that the fragmentation process in the periphery of G35.6 should occur between 1.6 and 5.3 Myr after its formation, a later point in time than the G35.6 dynamical age derived above. The range in the fragmentation time is inferred by considering the error in the original ambient density and the range in turbulent velocity. Thus, we conclude that the formation of the YSOs lying at the border of the HII region most probably results from other processes, such as the radiative driven implosion (RDI) mechanism, which consists of interactions of the ionization front with pre-existing condensations (Lefloch \& Lazareff 1994), or smallscale Jeans gravitational instabilities in the collected layer.

\section{Summary}

Using multiwavelength surveys and archival data, we have studied the ISM towards the HII region G35.673-00.847 (G35.6). This work is part of a systematic study that we are performing to increase observational evidence of triggered star formation in the surroundings of HII regions. The main results can be summarized as follows:

(a) The PAH emission around G35.6 seen at $8 \mu \mathrm{m}$ shows that the HII region has an almost semi-ring like shape with a cut towards the Galactic west. The radius of this semi-ring is about 1.5. The $24 \mu \mathrm{m}$ emission reveals the presence of hot dust in the interior of the HII region.

(b) The radio continuum emission indicates that towards the south of G35.6, also identified as NVSS 185929+020334, lies the radio source NVSS $185938+020012$, which is probably another HII region. From the HI absorption analysis, we conclude that both sources are located at the same distance, and from the central velocity of the related molecular gas, we estimate that the whole complex is at the kinematic distance of $\sim 3.7 \mathrm{kpc}$.

(c) Using the ${ }^{13} \mathrm{CO} J=1-0$ transition, we analyzed the molecular gas around G35.6. We identified a molecular shell composed of clumps distributed around the HII region, suggesting that its expansion is collecting the material. The molecular shell has a density of about $10^{4} \mathrm{~cm}^{-3}$.

(d) From a photometric study and a SED analysis, we discovered several sources (YSO candidates) very likely embedded in the molecular shell.

(e) We have presented some indirect evidence of location and properties of the exciting star(s) of G35.6 and NVSS $185938+020012$. In the case of G35.6, from the radio continuum flux, the near-IR photometry, and the physical location of the analyzed sources, we find four candidates, likely O-type stars, to be the ionizing agent of the HII region. Among them, two are located close to the maximum of the $24 \mu \mathrm{m}$ emission, and one of them (our source \#6) appears to be within a hole of $5.8 \mu \mathrm{m}$ emission, implying that it is the most likely candidate. In the case of NVSS 185938+020012, we suggest that the exciting $\operatorname{star}(\mathrm{s})$ should be later than an O9.5V star.

(f) Analyzing the HII region G35.6 dynamical age and the fragmentation time of the molecular shell surrounding the HII region, we exclude collect and collapse being the mechanism responsible for the YSO formation. We propose other possible processes of formation, such as radiative-driven implosion and/or small-scale Jeans gravitational instabilities in the collected layer.

Acknowledgements. We wish to thank the anonymous referee whose comments and suggestions have helped to considerably improve the paper. S.P. is member of the Carrera del investigador científico of CONICET, Argentina. A.P. and M.O. are doctoral and postdoctoral fellows of CONICET, Argentina, respectively. This work was partially supported by Argentina grants awarded by UBA, CONICET and ANPCYT.

\section{References}

Allen, L. E., Calvet, N., D’Alessio, P., \& et al. 2004, ApJS, 154, 363

Bohlin, R. C., Savage, B. D., \& Drake, J. F. 1978, ApJ, 224, 132

Chaisson, E. J. 1976, in Frontiers of Astrophysics, 259

Cohen, M., Parker, Q. A., Green, A. J., et al. 2007, ApJ, 669, 343

Condon, J. J., Cotton, W. D., Greisen, E. W., et al. 1998, AJ, 115, 1693

Deharveng, L., Zavagno, A., \& Caplan, J. 2005, A\&A, 433, 565

Dyson, J. E., \& Williams, D. A. 1980, Physics of the interstellar medium (New York: Halsted Press), 204

Elmegreen, B. G., \& Lada, C. J. 1977, ApJ, 214, 725

Fazio, G. G., Hora, J. L., Allen, L. E., et al. 2004, ApJS, 154, 10

Fich, M., Blitz, L., \& Stark, A. A. 1989, ApJ, 342, 272

Gail, H., \& Sedlmayr, E. 1979, A\&A, 76, 158

Inoue, A. K. 2001, AJ, 122, 1788 
Inoue, A. K., Hirashita, H., \& Kamaya, H. 2001, ApJ, 555, 613

Jackson, J. M., Rathborne, J. M., Shah, R. Y., et al. 2006, ApJS, 163, 145

Kolpak, M. A., Jackson, J. M., Bania, T. M., Clemens, D. P., \& Dickey, J. M. 2003, ApJ, 582, 756

Lefloch, B., \& Lazareff, B. 1994, A\&A, 289, 559

Lockman, F. J. 1989, ApJS, 71, 469

Martins, F., \& Plez, B. 2006, A\&A, 457, 637

Martins, F., Schaerer, D., \& Hillier, D. J. 2005, A\&A, 436, 1049

Neckel, T., \& Klare, G. 1980, A\&AS, 42, 251

Petriella, A., Paron, S., \& Giacani, E. 2010, A\&A, 513, A44

Pomarès, M., Zavagno, A., Deharveng, L., et al. 2009, A\&A, 494, 987

Reich, W., Fuerst, E., Haslam, C. G. T., Steffen, P., \& Reif, K. 1984, A\&AS, 58, 197

Rieke, G. H., \& Lebofsky, M. J. 1985, ApJ, 288, 618
Robitaille, T. P., Whitney, B. A., Indebetouw, R., Wood, K., \& Denzmore, P. 2006, ApJS, 167, 256

Robitaille, T. P., Whitney, B. A., Indebetouw, R., \& Wood, K. 2007, ApJS, 169, 328

Robitaille, T. P., Meade, M. R., Babler, B. L., et al. 2008, AJ, 136, 2413

Simon, R., Jackson, J. M., Clemens, D. P., Bania, T. M., \& Heyer, M. H. 2001, ApJ, 551, 747

Stil, J. M., Taylor, A. R., Dickey, J. M., et al. 2006, AJ, 132, 1158

Werner, M. W., Roellig, T. L., Low, F. J., et al. 2004, ApJS, 154, 1

Whitworth, A. P., Bhattal, A. S., Chapman, S. J., Disney, M. J., \& Turner, J. A. 1994a, A\&A, 290, 421

Whitworth, A. P., Bhattal, A. S., Chapman, S. J., Disney, M. J., \& Turner, J. A. 1994b, MNRAS, 268, 291

Zavagno, A., Pomarès, M., Deharveng, L., et al. 2007, A\&A, 472, 835 